\title{
Evaluation of Adherence to Ambulatory Liquid Oxygen Treatment: Are Commercialized Dual-Pressure Transducers Helpful?
}

\author{
Mafalda van Zeller MD, Ermelinda Eusébio, João Almeida MD, \\ and João Carlos Winck MD PhD
}

\begin{abstract}
BACKGROUND: Treatment adherence is widely recognized as a critical problem in long-term oxygen therapy, particularly in ambulatory liquid oxygen (LOX) systems. Adherence-monitoring strategies may be helpful in managing patients. We evaluated subjects' adherence to LOX using VisionOx and compared these results with the subjects' own adherence diaries and self-reported perceptions of use. METHODS: Patients using LOX were recruited for a clinical interview; the number of days/week and the mean time of use according to subjects' perceptions were recorded. A 14-day diary was provided for every subject while VisionOx was attached to the LOX. VisionOx is a small device that uses pressure transducers to detect oxygen flow and the subject's breathing frequency. Information is stored and downloaded using dedicated software. RESULTS: Nineteen subjects were included $(57.9 \%$ male with a median age of 63 years). When asked about the perception of LOX use, subjects self-reported using the device for a median of $100.0 \%$ of days $\mathbf{7 8 . 9 \%}$ reported to have used it every day) for a median time of $180 \mathrm{~min} /$ day. According to data from VisionOx and subjects' diaries during the 14-day evaluation period, the median use was $92.8 \%$ of days for $210 \mathrm{~min} /$ day. No difference was found between the diaries and VisionOx data. Regarding subjects' perceptions of use, the declared use of LOX percent was significantly higher than reported in the diaries $(P=.045)$ and VisionOx monitoring $(P=.045)$ even though both underestimated the median use per day. CONCLUSIONS: Subjects overestimated adherence to LOX therapy (when measuring percent of days of use) compared to adherence diary and objective adherence monitoring. Because no significant difference was found comparing the diaries and VisionOx use, either may be helpful in clinical practice. Key words: ambulatory liquid oxygen; adherence; VisionOx. [Respir Care 2014;59(9):1376-1380. (C) 2014 Daedalus Enterprises]
\end{abstract}

\section{Introduction}

Ambulatory oxygen is delivered by equipment that can be carried by patients during exercise and the activities of daily life. ${ }^{1}$ It allows patients with severe respiratory diseases to remain active within the community and to maintain exercise tolerance and social interactions. ${ }^{2}$

Recently, $\mathrm{Criner}^{3}$ reviewed the end points for supplemental oxygen at home, such as mortality, effects on func-

Drs van Zeller and Almeida and Ms Eusébio are affiliated with the Department of Pulmonology, Centro Hospitalar de São João, Porto, Portugal. Dr Winck is affiliated with the Department of Pulmonology, Faculty of Medicine, University of Porto, Porto, Portugal, and Linde Healthcare, Pullach, Germany.

Dr Winck is currently an employee of Linde AG, which is the proprietor of VisionOx. The other authors have disclosed no conflicts of interest. tional performance, sensation of dyspnea, cognitive function, and quality of life, and highlighted areas where future research is needed. With technological improvements, patients have more practical, simple, and smaller ambulatory oxygen systems, allowing for easier outdoor use.

Specific criteria should be followed when prescribing ambulatory oxygen as an addition to long-term oxygen

\footnotetext{
A version of this paper was presented by Mafalda van Zeller at the European Respiratory Society Annual Congress, held September 7-11, 2013, in Barcelona, Spain.

Correspondence: Mafalda van Zeller MD, Department of Pulmonology, Centro Hospitalar de São João, Alameda Hernani Monteiro, 4200-319 Porto, Portugal. E-mail: vanzeller.mafalda@gmail.com.
}

DOI: $10.4187 /$ respcare. 02833 
therapy or for exercise desaturation in patients who are normoxic at rest. Patient adherence to supplemental oxygen is variable and often unpredictable. ${ }^{3}$

Adherence to prescribed medication is important to optimize the management of long-term conditions. ${ }^{4}$ In fact, Lacasse et $\mathrm{al}^{5}$ had to prematurely end a trial investigating the use of ambulatory oxygen because the participants were not adherent, which is wasteful of health resources. ${ }^{2}$

Determining the liquid oxygen (LOX) treatment compliance is important and enables the creation of mechanisms aimed at improving adherence or supporting the decision to suspend the treatment. This is particularly important at a time of financial austerity affecting health care services in Portugal as well as worldwide.

Mechanisms that allow physicians to recognize the actual adherence to prescribed therapy may lead to the development of enhanced adherence and compliance strategies, such as adapting the oxygen system to patients' desires.

Previous studies have defined adherence to ambulatory oxygen as the mean use of ambulatory oxygen $>30 \mathrm{~min} /$ days. ${ }^{6}$ However, this number is arbitrary, and there are no clear data regarding the optimal adherence to LOX. Patients should use LOX during physical activity and time spent outdoors, and patients should engage in both as often as possible.

Strategies to evaluate patients' use of LOX are necessary. We aimed to evaluate patient adherence by examining and comparing self-reported adherence, adherence diaries, and electronic monitoring, which to our knowledge have never been used for the evaluation of ambulatory oxygen adherence evaluation.

\section{Methods}

\section{Study Design}

This is a prospective observational study approved by the hospital's ethical committee. All of the subjects provided consent for the study. The study was developed and performed in the Department of Pulmonology, Centro Hospitalar de São João, Porto, Portugal.

All eligible subjects were prescribed ambulatory LOX systems at our center, and the LOX systems were provided by a single home care company (Linde Healthcare). None of the subjects had experienced an exacerbation during the previous 4 weeks. After the subjects were identified in the hospital database, they were recruited by a resident. In the clinic, we collected subjects' demographics, respiratory diagnosis, and information regarding when any respiratory domiciliary care had been prescribed.

The number of days/week and the median time of LOX use according to subjects' self-reports were registered. Later, an adherence diary was provided for 14 days (Fig. 1), in which subjects had to register the number of times per day LOX was used and also the duration of LOX use. VisionOx (Fig. 2) was attached to the LOX (Fig. 3). VisionOx is a small

\section{QUICK LOOK}

\section{Current knowledge}

Ambulatory home oxygen therapy improves quality of life and mobility and reduces mortality in patients with COPD and hypoxemia on room air. Compliance with home oxygen prescriptions is poor owing to complexity and equipment issues.

What this paper contributes to our knowledge.

Subjects overestimated adherence to liquid oxygen (LOX) therapy compared to an adherence diary and objective adherence monitoring. No significant difference was found between using a diary or an electronic monitoring system. Either method may be helpful in clinical practice to monitor adherence to LOX therapy.

device that uses pressure transducers to detect oxygen flow and the subject's breathing frequency. This information is stored and then downloaded using dedicated software.

Subjects were informed that the main objective of VisionOx was to record their actual oxygen flow.

\section{Statistical Analysis}

Descriptive statistics were generated for the study sample using proportions for categorical variables and median with interquartile range as well as means \pm SD for quantitative variables.

Because the hypothesis of a normal distribution of these parameters was rejected using the Shapiro-Wilk test and the sample size was small, further analysis was conducted using nonparametric tests.

The results for subjects' adherence to LOX obtained with the different methods were compared using the related-samples Friedman's 2-way analysis of variance by rank and pair-wise comparisons. To evaluate the consistency of the results obtained by the 2 measurement techniques, a Pearson correlation test was performed, and $r^{2}=0.70$ or higher was considered a strong positive relationship.

To evaluate accurate reports, categorical variables ( $>90 \%,<90 \%$ of use per week) were created to calculate the kappa measure of agreement.

Statistical analyses were performed using the software SPSS 21. The level of significance was fixed at .05.

\section{Results}

According to the hospital reimbursement database, there were 101 patients with LOX prescriptions when the study started. Thirty-one patients were eligible for the study according to the inclusion criteria. From the identification process to the invitation for the clinical interview, 5 pa- 


\section{Liquid Oxygen Systems (LOX) - Daily Diary}

Name:

Oxygen Flow (L/min):

Date: to

\begin{tabular}{|l|c|c|c|c|c|c|}
\hline & $\begin{array}{c}\text { How many } \\
\text { times did you } \\
\text { use LOX? }\end{array}$ & $\begin{array}{c}\text { For how long } \\
\text { did you use } \\
\text { LOX outdoors? }\end{array}$ & $\begin{array}{c}\text { For how long } \\
\text { did you use } \\
\text { LOX indoors? }\end{array}$ & $\begin{array}{c}\text { Was LOX } \\
\text { helpful on } \\
\text { daily } \\
\text { activities? } \\
\text { (Yes/No) }\end{array}$ & $\begin{array}{c}\text { Was LOX } \\
\text { helpful } \\
\text { controlling } \\
\text { dyspnoea? } \\
\text { (Yes/No) }\end{array}$ & Comments \\
\hline Monday & & & & & & \\
\hline Tuesday & & & & & &
\end{tabular}

Fig. 1. Diary for recording adherence to liquid oxygen (LOX).

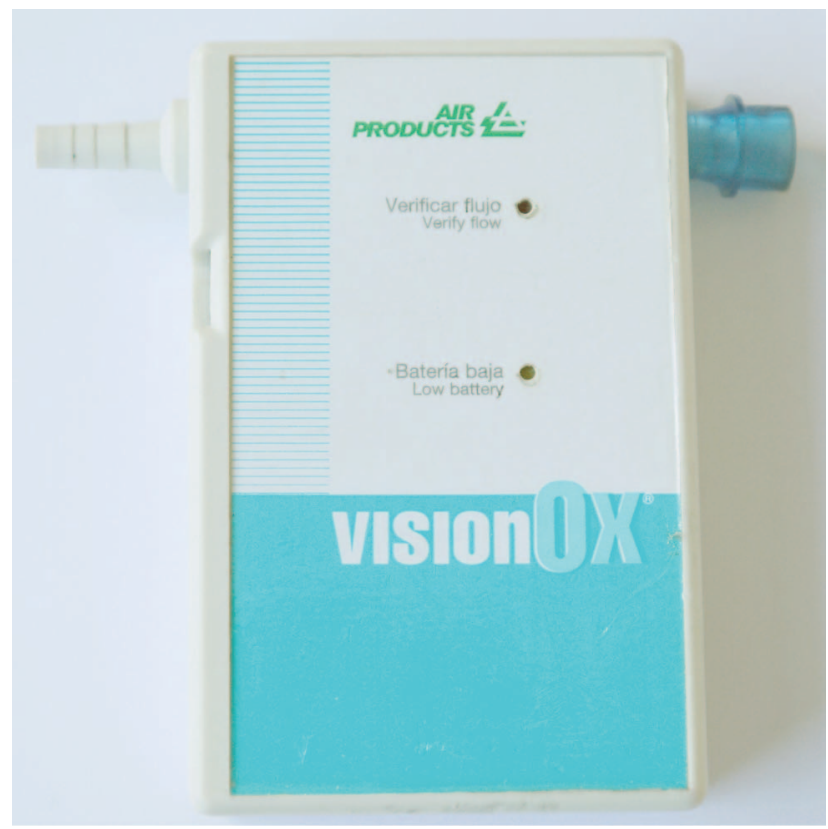

Fig. 2. VisionOx.

tients died, 4 were denied prescription renewal by their physician for lack of activity or adherence issues, 2 were out of the country for evaluation in a transplant center, and one refused to participate. Only 19 subjects were included, and their characteristics are described in Table 1.

Subjects' median time of schooling was 4 years; 4 $(21.1 \%)$ maintained a career while the others were retired, $6(31.6 \%)$ due to respiratory disease.

Subjects used LOX for a mean of $3.26 \pm 2.75$ years, and the mean time between the respiratory diagnosis and LOX prescription was $17.61 \pm 14.99$ years. All subjects received long-term oxygen therapy, and the majority were

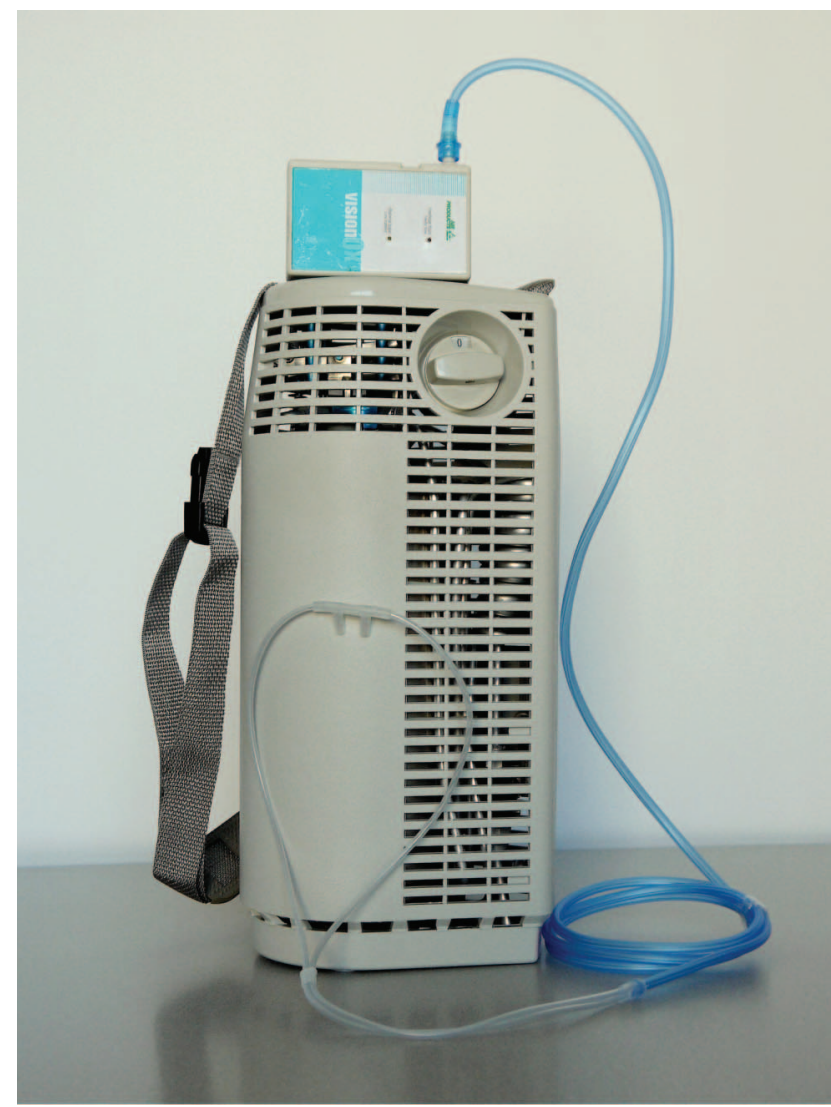

Fig. 3. VisionOx attached to a liquid oxygen system.

also prescribed noninvasive ventilation; $4(21.1 \%)$ were previously given noninvasive ventilation, and $6(31.6 \%)$ were given it simultaneously with LOX.

When asked about their adherence to LOX (Table 2), subjects reported a median use of $100 \%$ of days. The 
Table 1. Subjects' Characteristics

\begin{tabular}{lcc}
\hline \hline \multicolumn{1}{c}{ Characteristics } & Median & IQR \\
\hline Age (y) & 63 & $56-68$ \\
Years of education & 4 & $4-4$ \\
Time of LOX prescription (y) & 2 & $1-4$ \\
& $n$ & \\
Sex & & \\
Male & 11 & 57.9 \\
Female & 8 & 42.1 \\
Respiratory diagnosis & & \\
COPD & 8 & 42.1 \\
Bronchiectasis & 4 & 21.1 \\
Hypersensitivity pneumonitis & 2 & 10.5 \\
Tuberculosis sequelae & 1 & 5.3 \\
Asthma and tuberculosis sequelae & 1 & 5.3 \\
Silicosis & 1 & 5.3 \\
Idiopathic pulmonary fibrosis & 1 & 5.3 \\
Lymphangioleiomyomatosis & 1 & 5.3 \\
Total & 19 & 100 \\
& & \\
IQR = interquartile range & & \\
LOX = liquid oxygen & & \\
\hline
\end{tabular}

Table 2. Adherence to Liquid Oxygen Treatment

\begin{tabular}{lccc}
\hline \hline & \multicolumn{3}{c}{ Method Used } \\
\cline { 2 - 4 } & Questionnaire & $\begin{array}{c}\text { Subject } \\
\text { Daily Diary }\end{array}$ & VisionOx \\
\hline Time of use/day (min) & 180 & 210 & 210 \\
$\quad$ Median & 120 & 165 & 150 \\
P25 & 300 & 375 & 300 \\
P75 & & & \\
\% of days of use/week & 100 & 92 & 92 \\
$\quad$ Median & 100 & 38 & 42 \\
P25 & 100 & 100 & 100 \\
P75 & & & \\
& & & \\
P25 = 25th percentile & & & \\
P75 = 75th percentile & & & \\
\hline
\end{tabular}

majority of subjects (78.9\%) reported daily use, for a median time of $180 \mathrm{~min} /$ day.

No technical problems were found with VisionOx, and all of the information was successfully downloaded.

During the 14 days of evaluation, the results according to the diaries and VisionOx were similar, with a median use of $92.8 \%$ of days for $210 \mathrm{~min} /$ days. No difference was found between the diaries and the VisionOx data.

The percent of days of use as declared by the subjects was significantly higher than that indicated by the diaries and VisionOx $(P=.045)$ monitoring, although the mean time of use per day was underestimated (180 vs $210 \mathrm{~min}$ ).
We found a strong Pearson correlation $(\mathrm{r}=0.81$, $P=.001$ ) between the data collected in the diaries and the data from electronic monitoring, but when compared to subjects' perceptions, no relationship was found $(\mathrm{r}=0.22$, $P=.40 ;$ and $\mathrm{r}=0.42, P=.87$, respectively).

The percent of days of use was analyzed using categorical variables $(<90 \%$ and $\geq 90 \%)$ as determined in methods . We calculated the kappa measure of agreement between the diaries and VisionOx results, confirming a good correlation (kappa 0.89, $P=.001$ ).

According to the VisionOx data, LOX use of $>80 \%$ of days was found to be significantly higher in male subjects $(P=.02)$, but no relationship was found regarding age $(P=.34)$, level of education $(P=.57)$, time of LOX use $(P=.40)$, LOX system model or weight $(P=.24)$, and distance walked on a 6-min walk test $(P=.75)$.

\section{Discussion}

This study evaluated subjects' adherence to LOX and compared subjects' perceptions of use with an adherence diary and VisionOx, a commercialized dual-pressure transducer.

The full benefit of medications can be achieved only if patients adhere to prescribed treatment regimens. ${ }^{7}$ However, among patients with chronic illnesses, $\sim 50 \%$ do not take medications as prescribed. ${ }^{8}$ Adherence to therapy varies in different diseases, and chronic conditions present particular difficulties for medication adherence and persistence. ${ }^{9}$ The treatment regimens are relevant. Once-a-day oral medications are more appealing than eye drops, topical or inhaler treatments, and nocturnal oxygen therapy. ${ }^{8-11}$

Self-reporting is a frequently used method for the assessment of medication compliance behavior in patients with chronic illness. While self-reporting is an easy and practical method, a subject's memory of past events and reliability is variable. Additionally, subjects may attempt to please the researcher or clinician. ${ }^{12}$ In fact, self-reporting has been characterized as "notoriously unreliable," overestimating true compliance by $\sim 30 \% .{ }^{13}$ Efforts to reduce the reliance on memory have led to the use of medication diaries. Nevertheless, reliability of these data is questionable. Thus, the electronic monitoring of medication use has become the accepted standard for validating medication adherence in diseases. ${ }^{14,15}$ In respiratory diseases, the electronic monitoring of inhaled and nebulized medication has been shown to provide a more precise measurement of long-term medication use than self-reporting on diary cards. ${ }^{12,16}$ Noninvasive ventilation uses an electronic monitoring of treatment adherence that can be easily downloaded for research and for daily evaluation to adjust treatment and therapy goals.

We found that our subjects used LOX for an acceptable percentage of days per week, and men seemed to use LOX more frequently than women. Our subjects used LOX for reasonable periods of time per day (median $210 \mathrm{~min}$ ) com- 
pared to the mean use of ambulatory oxygen of $40 \pm 36 \mathrm{~min} /$ day described in other studies that used questionnaires. ${ }^{6}$

Subjects' perceptions did not seem reliable because they overestimated the frequency of LOX use, while both VisionOx and the diaries were more accurate. Interestingly, although subjects overestimated the percent of days of use, they underestimated the median time of use per day. Perhaps subjects felt compelled to please the physician conducting the clinical interview by reporting almost daily LOX use.

No statistically significant difference was found between this device and the subjects' daily diaries, perhaps because subjects knew that their LOX was being monitored. In addition, subjects used the diaries for only 14 days and were highly motivated because the diaries were noted to be highly relevant during the clinical interviews.

The limitations of our study include our small number of subjects and that VisionOx could not be attached to the LOX without subject knowledge. Although subjects were informed that the device was being used to determine actual oxygen flow and not adherence, the presence of the Hawthorne effect (commonly referred to as the observerexpectancy effect) cannot be excluded. Additionally, the electronic device provided information regarding LOX use but not subject activity. Pedometers were not used. Therefore, we had to rely on subject information to evaluate whether LOX was used during outdoor activities.

The knowledge of real patients' adherence is essential in clinical practice because it will enable us to develop strategies $^{17}$ to improve compliance or to support a decision to discontinue the therapy. According to previous studies, a lack of information, fear that the oxygen might be depleted, embarrassment, and the weight of the ambulatory oxygen system are the main barriers to patient adherence. ${ }^{2}$ The adequacy of ambulatory oxygen prescription in improving patients' quality of life has been discussed previously in studies using activity monitors, ${ }^{18,19}$ but to our knowledge, patients' adherence to ambulatory LOX has never been evaluated using electronic monitoring.

Commercialized dual-pressure transducers, such as VisionOx, have been used to evaluate patients' adherence to home-use oxygen, but they can also offer other possibilities, including registering patients' breathing frequency and oxygen flow. Yañez et al used VisionOx to monitor respiratory frequency in subjects with COPD who were receiving domiciliary oxygen therapy and found a significant increase in breathing frequency days before subjects required hospitalization for COPD exacerbation, suggesting that monitoring may be used for early intervention. ${ }^{20}$

In our study, subjects' perceptions of use were not reliable. Similarly, in noninvasive ventilation, information regarding LOX adherence using VisionOx may be easily downloaded, and strategies to optimize LOX therapy can be developed only if adherence is acknowledged as a limitation.
In conclusion, subjects overestimated the frequency of LOX use compared to the adherence found using an adherence diary and objective monitoring. Because no significant difference was found between the diary and VisionOx, both may be helpful in clinical practice.

\section{REFERENCES}

1. Ram FS, Wedzicha JA. Ambulatory oxygen for chronic obstructive pulmonary disease. Cochrane Database Syst Rev 2002;(2):CD000238

2. Arnold E, Bruton A, Donovan-Hall M, Fenwick A, Dibb B, Walker E. Ambulatory oxygen: why do COPD patients not use their portable systems as prescribed? A qualitative study. BMC Pulm Med 2011; 11:9. doi:10.1186/1471-2466-11-9.

3. Criner GJ. Ambulatory home oxygen: what is the evidence for benefit, and who does it help? Respir Care 2013;58(1):48-64.

4. Lehane E, McCarthy G. Medication nonadherence-exploring the conceptual mire. Int J Nurs Pract 2009;15(1):25-31.

5. Lacasse Y, Lecours R, Pelletier C, Bégin R, Maltais F. Randomised trial of ambulatory oxygen in oxygen-dependent COPD. Eur Respir J 2005;25(6): 1032-1038.

6. Marien H, Marchand E. Adherence to ambulatory oxygen therapy. Assessment and determinants in patients receiving long-term oxygen therapy. Rev Mal Respir 2011;28(7):873-880.

7. Osterberg L, Blaschke T. Adherence to medication. N Engl J Med 2005;353(5):487-497.

8. De Geest S, Sabaté E. Adherence to long-term therapies: evidence for action. Eur J Cardiovasc Nurs 2003;2(4):323

9. Yeaw J, Benner JS, Walt JG, Sian S, Smith DB. Comparing adherence and persistence across 6 chronic medication classes. J Manag Care Pharm 2009;15(9):728-740.

10. Sabaté E, ed. Adherence to long-term therapies: evidence for action. Geneva, Switzerland: World Health Organization; 2003.

11. Restrepo RD, Alvarez MT, Wittnebel LD, Sorenson H, Wettstein R, Vines DL, et al. Medication adherence issues in patients treated for COPD. Int J Chron Obstruct Pulmon Dis 2008;3(3):371-384.

12. Berg J, Dunbar-Jacob J, Rohay JM. Compliance with inhaled medications: the relationship between diary and electronic monitor. Ann Behav Med 1998;20(1):36-38

13. Anonymous. Are you taking the medicine? Lancet 1990;335(8684): 262-263.

14. Cramer JA. Microelectronic systems for monitoring and enhancing patient compliance with medication regimens. Drugs 1995;49(3):321-327.

15. Choo PW, Rand CS, Inui TS, Lee ML, Cain E, Cordeiro-Breault M, et al. Validation of patient reports: automated pharmacy records and pill counts with electronic monitoring of adherence to antihypertensive therapy. Med Care 1999;37(9):846-857.

16. Butz AM, Donithan M, Bollinger ME, Rand C, Thompson RE. Monitoring nebulizer use in children: comparison of electronic and asthma diary data. Ann Allergy Asthma Immunol 2005;94(3):360-365.

17. Walshaw MJ, Lim R, Evans CC, Hind CR. Factors influencing the compliance of patients using oxygen concentrators for long-term home oxygen therapy. Respir Med 1990;84(4):331-333.

18. Peckham DG, McGibbon K, Tonkinson J, Plimbley G, Pantin C. Improvement in patient compliance with long-term oxygen therapy following formal assessment with training. Respir Med 1998;92(10):12031206.

19. Sandland CJ, Morgan MD, Singh SJ. Patterns of domestic activity and ambulatory oxygen usage in COPD. Chest 2008;134(4):753-760.

20. Yañez AM, Guerrero D, Pérez de Alejo R, Garcia-Rio F, Alvarez-Sala JL, Calle-Rubio M, et al. Monitoring breathing rate at home allows early identification of COPD exacerbations. Chest 2012;142(6):1524-1529. 